\title{
GESTIÓN DE LA INVESTIGACIÓN EN LA UNIVERSIDAD RICARDO PALMA (RETOS Y DESAFÍOS)
}

\author{
Hugo SÁNCHEZ CARLESSI \\ Vicerrector de Investigación \\ Universidad Ricardo Palma \\ hector.sanchez@urp.edu.pe
}

\section{RESUMEN}

Desde el año 1969, en que se establece la Ley Orgánica de la Universidad Peruana (DL. Nº 17437), hasta el presente, han pasado 48 años. Observándose, que, las universidades del país muy poco se preocuparon por inculcar la investigación en los estudios de pregrado. Se fue anulando el trabajo de tesis para el bachillerato y el título. En sí, autoridades y alumnos, muy poco se interesaron por la investigación científica o tecnológica. Y, esto repercutió, en los estudios de maestría y doctorado en donde el eje curricular es la investigación. No obstante, la Universidad Ricardo Palma a través del Centro de Investigación entre los años 1996 al 2015, y en cada Facultad, logró apoyar de algún modo, a los profesores investigadores interesados en desarrollar sus proyectos de investigación. Al crearse, la Ley 30220 y, constituirse el Vicerrectorado de Investigación, la gestión se encontró con algunos problemas y, limitaciones académico administrativos propios de la falta de recursos. Esto, hace plantear, el reto de sacar adelante la investigación en la URP en concordancia con las evaluaciones diseñadas por el Licenciamiento institucional y la acreditación. Lo que ha permitido, tener notorios logros entre los años 2016 y 2017, en los aspectos: normativos, organizativos, de financiamiento, capacitación en investigación, publicaciones, investigación formativa, artículos científicos, profesores investigadores de la URP, inscritos en DINA y REGINA y, en la creación del Repositorio Institucional.

\section{PALABRAS CLAVE}

Gestión en investigación, desarrollo científico, Universidad Ricardo Palma.

\section{MANAGEMENT OF RESEARCH AT THE RICARDO PALMA UNIVERSITY (CHALLENGES AND DEFIANCE)}

\begin{abstract}
Since 1969, when the Organic Law of the Peruvian University (DL N ${ }^{\circ}$. 17437) up to the present, 48 years have passed. Being observed, that, the universities of the country very little worried about instilling the research in the undergraduate studies. Skillfully, the thesis exploration was canceled. In themselves, authorities and students, very little was interested in scientific or technological research. And, this affected, in the studies of masters and doctorate where the curricular axis is the investigation. However, the Ricardo Palma University through the Research Center between 1996 and 2015, and in each Faculty, managed to support in some way, the research professors interested in developing their research projects. When the Law 30220
\end{abstract}


was created, and the Office of the Vice-Rector for Research was established, management encountered some problems and academic administrative limitations inherent in the lack of resources. This, makes raise, the challenge to take ahead the investigation in the URP in agreement with the evaluations designed by the institutional Licensing and the accreditation. What has allowed to have notable achievements between 2016 and 2017, in the normative, organizational aspects, financing, training in research, publications, formative research, scientific articles. And, as regards, URP research professors, registered in DINA and REGINA and, in the presence of the Institutional Repository.

\section{KEYWORDS}

Research management, scientific development, Ricardo Palma University

Recibido: $5 / 9 / 2017$

Aprobado: 10/11/2017 


\section{Antecedentes}

El reconocimiento y presencia de la investigación en la Universidad Ricardo Palma (URP), no es nuevo. El primer avance se dio luego de la creación de la URP en el año 1968, por el gobierno militar del General Juan Velasco Alvarado. La reforma educativa peruana iniciada, se extiende al nivel universitario y en el año 1969 se da la Ley Orgánica de la Universidad Peruana (DL.N 17437), con la cual se organiza el sistema departamentalista en cada universidad, y se reconoce la importancia de la investigación como una función básica de la Universidad Peruana. En este marco en la URP se crea la Dirección Universitaria de Investigación.

Varios años después, ya con la presencia del gobierno democrático del Arq. Fernando Belaunde Terry se da la Ley Universitaria № 23733 en el año 1983. En las universidades se retorna al sistema facultativo y se destaca la investigación como una de los fines básicos de la Universidad (art 20). En la URP se crea el Centro de Investigación de la URP (CIURP), y en las facultades se crean los institutos de investigación, y a nivel central se van fundando los institutos de investigación especializados dependientes del Rectorado.

El 07 de mayo de 1993 se aprueba el primer documento normativo de política y líneas de investigación, por R.R 930554-SG. y por R.R N 930550 del mismo día igualmente se aprueba el Reglamento del Centro de Investigación de la URP. Entre los años 1991 a 1995 se realizaron diversas investigaciones a través de los institutos de investigación de las diversas facultades mucha de ellas en forma asistemática y los resultados fueron publicados en las revistas de cada Facultad. Predominó, en gran parte, la motivación, interés y esfuerzo personal del docente investigador de la URP, ya que el apoyo económico era muy limitado.

En el año 1996 el Centro de Investigación de la URP revisó el reglamento vigente de la época, y elaboró un nuevo reglamente de investigación de la URP que quedó pendiente de aprobación. Ese mismo año, se da el primer impulso para que los docentes de las distintas facultades realizaran investigación universitaria. Se presentaron 49 proyectos de investigación a los cuales se les dio financiamiento a través del CIURP. Este apoyo económico a los proyectos se ha mantenido a través de los años aunque no con las cantidades necesarias para cubrirlas plenamente. (Ver Cuadro $\mathrm{N}^{\circ} 1$ )

Cuadro N 01. Números de proyectos: 1996 - 2015

\begin{tabular}{|l|l|l|l|l|l|l|l|l|l|}
\hline 1996 & 1997 & 1998 & 1999 & 2000 & 2001 & 2002 & 2003 & 2004 & 2005 \\
\hline 49 & 28 & 11 & 21 & 13 & 21 & 25 & 14 & 27 & 23 \\
\hline
\end{tabular}

\begin{tabular}{|l|l|l|l|l|l|l|l|l|l|}
\hline 2006 & 2007 & 2008 & 2009 & 2010 & 2011 & 2012 & 2013 & 2014 & 2015 \\
\hline 25 & 32 & 11 & 18 & 14 & 14 & 16 & 19 & 15 & 19 \\
\hline
\end{tabular}

Fuente: Documentos, boletines y publicaciones CIURP: 1996-2015 Elaboración: Propia.

Fueron las facultades de Psicología, Biología y Humanidades las que a través de los años demostraron tener mayor presencia en cuanto a proyectos de investigación presentados por los docentes. Hasta el año 2015 entre los hitos más resaltantes de la investigación en la URP figuran: 
a) Desde sus inicios la Carrera de Psicología de la URP ha mantenido en su plan de estudios las asignaturas de Taller de Investigación I y II, las que se mantienen hasta la actualidad y que hizo posible que la Facultad de Psicología cuente con una cantidad significativa de egresados con Tesis profesional.

b) En el año 1993 la Facultad de Psicología publicó la obra Resúmenes de Tesis 19871993, conteniendo los resúmenes de las tesis presentadas por los bachilleres desde el año 1987 hasta el año 1993.

c) En los años 1995 y 1996 La facultad de Psicología publicó la Revista del Instituto de Investigaciones de la Facultad. En esta última se presentaban los informes de investigación realizados siguiendo el esquema del modelo APA.

d) En el año 1996 a través del CIURP se realizó el I Programa de Capacitación docente en Investigación científica y tecnológica con participación de 63 docentes de la URP.

e) En el año 1998 el CIURP publica la Revista Scientia, publicación periódica anual que tiene presencia y que va por su volumen № 18.

f) En el año 2002 la Escuela de Pos grado de la URP realizó el I Congreso Nacional de Escuelas de Pos Grado de la Universidad Peruana, donde se presentaron muchos trabajos de investigación y artículos académicos vinculados con la investigación.

g) En el año 2013 la Facultad de Psicología de la URP publica la obra Resúmenes de Tesis 1987-2012.

h) En el año 2014 después de 31 años de vigencia de la Ley 23733 se da la Ley Universitaria actual $N^{\circ} 30220$ que en sus artículos $3^{\circ}$ y $7^{\circ}$ considera como función básica de la universidad investigar, para lo cual deben crearse $y / 0$ adecuarse los órganos y dependencias respectivas a nivel central y a nivel de la escuela de posgrado, facultades y escuelas profesionales. En la URP se crea el Vicerrectorado de Investigación, continúa el CIURP como órgano de ejecución del VRI, se redefinen las unidades e institutos de investigación en las Facultades y Escuela de Posgrado. El Vicerrectorado de investigación comienza a operar a partir de diciembre del año 2015 al elegirse por primera vez al Vicerrector de Investigación.

\section{Problemas y limitaciones al inicio de la gestión}

Pasamos a reseñar los principales problemas organizativos, metodológicos, técnicoadministrativos y culturales que se presentaron al iniciarse la gestión del Vicerrectorado de Investigación en el marco de la Ley Universitaria № 30220.

\section{Primero: Apoyo prioritario por un campo específico de investigación.}

De acuerdo a lo normado, la investigación en las universidades se debe dar en los campos científicos, tecnológicos y humanísticos, sin embargo debemos reconocer que los órganos promotores dan más apoyo económico al campo tecnológico y a las llamadas ciencias duras, tales como la física, la química, la biología, la matemática y desestiman o consideran en segundo plano a las llamadas ciencias blandas, en especial las ciencias, sociales, humanidades. La URP es una universidad definidamente humanista y si bien tiene carreras profesionales relacionadas con la ciencia y la tecnología, muchas de sus investigaciones se perfilan en el campo humanístico.

De igual forma se da más importancia a la tecnología relacionada con la informática, las telecomunicaciones, la electrónica, la mecatrónica, etc. y se deja en un segundo plano 
a las tecnologías que emplean otras disciplinas y carreras profesionales, tales como la tecnología educativa, la tecnología psicológica, la tecnología que se emplea en las ciencias sociales, entre otras. Todo esto limita al investigador y a la universidad lograr fuentes de financiamiento externo.

Segundo: El dilema de ejecutar investigaciones que contribuyan estrictamente al desarrollo científico o tecnológico o investigaciones socialmente necesarias.

La situación real de nuestro país nos inclina por el desarrollo de investigaciones aplicadas que sean útiles para nuestra sociedad y que contribuyan realmente al desarrollo del país antes que a investigaciones básicas orientadas al desarrollo de la ciencia y la tecnología "per se". Es sumamente difícil que nuestro País pueda desarrollar ciencia básica o proyectos de investigación tecnológica con los recursos que dispone. Es más viable realizar investigación aplicada socialmente necesaria e innovaciones tecnológicas.

\section{Tercero: Escasa o limitada capacitación y/o perfeccionamiento teórico-práctico de los profesores de la URP en materia de investigación.}

Por la historia de investigación en la URP se ha observado que son pocos los docentes que hacen investigación, mayoritariamente a los profesores les falta la capacitación metodológica y tecnológica adecuadas para elaborar sus proyectos de investigación sobre todo utilizando los medios informáticos y plataformas virtuales. La formación en investigación del docente es pobre. En el mundo universitario se observa que los planes de estudio de las diversas carreras profesionales si bien consideran la enseñanza de la investigación, sin embargo muchas veces estas asignaturas son dictadas por docentes que saben de investigación por lo que dicen los libros, pero nunca han realizado un proyecto de investigación. Lamentablemente los años del Bachillerato automático excluyeron a muchos profesionales como futuros investigadores.

\section{Cuarto: Imprecisión o falta de claridad y definición de los tipos y métodos de investigación.}

El desconocimiento, ambigüedad o imprecisión respecto a qué tipo de investigación se va a emplear para un proyecto específico puede afectar la política, los propósitos, los objetivos, el diseño específico de investigación y por tanto los resultados esperados. En relación a los métodos de investigación es importante resaltar que también la imprecisión o escasa comprensión de un método de investigación puede afectar los diseños y por tanto la validez, los alcances y los resultados de una investigación. La razón es que el método está articulado con un paradigma epistemológico asumido por el investigador. Por ejemplo, asumir el paradigma del positivismo lógico (que se ha generalizado para toda investigación cuantitativa), o asumir el paradigma comprensivo y hermenéutico que define la investigación cualitativa. Más aún, el problema se complica si los estudios pueden hacer uso de variados métodos que pueden ser ubicar entre los métodos teóricos y los métodos empíricos.

\section{Quinto: Escaso impulso a la investigación en el pregrado,}

Si contamos desde la época de la reforma universitaria (1968) hasta el presente son 47 años y, se observa que mayormente las universidades muy poco se han preocupado por inculcar la investigación en el pregrado. La eliminación del Bachillerato por tesis y el 
desplazamiento de la alternativa de Tesis para la licenciatura trajeron como consecuencia que las autoridades y por tanto los alumnos muy poco se interesaran por la investigación ni mucho menos por la tesis. Esta deficiencia se observa en el posgrado cuando en la maestría el alumno encuentra muchas dificultades para formular su proyecto de investigación para la tesis de grado. Esta deficiencia de hecho repercute en los estudios de doctorado en la que la investigación es mucho más exigente.

\section{Sexto: Ausencia de políticas claras y de líneas prioritarias de investigación en el posgrado.}

Por lo general, las universidades no han tenido una política ni línea definida en materia de investigación que pudieran servir de guía a los docentes y, alumnos para la selección de un tema y problema de investigación. Cada alumno o profesor investigador seleccionaba un tema de investigación más en función a sus intereses personales y sus posibilidades reales de realización del trabajo de investigación.

\section{Séptimo: Dificultad y limitaciones en la búsqueda de fuentes de financiamiento} para la investigación.

Si bien la URP ha reconocido la importancia de la investigación desde sus inicios, las previsiones económicas para su financiamiento siempre fueron muy limitadas y los apoyos económicos igualmente fueron limitados o restringidos.

\section{Avances y logros del Vicerrectorado de Investigación, 2016-2017}

A partir del año 2016 hasta el presente el Vicerrectorado de Investigación destaca los principales logros alcanzados:

\subsection{En el aspecto organizativo:}

Se ha definido la estructura organizativa de la Investigación en la URP, conformada por:

- El Vicerrectorado de Investigación como órgano de promoción, dirección, supervisión y control y el CIURP como órgano de ejecución del VRI.

- Determinación de las Unidades de Investigación de cada Facultad. Algunas conforman institutos de investigación.

- $\quad$ Determinación de la Unidad de Investigación de la Escuela de Posgrado.

- $\quad$ Además se cuenta con los institutos especializados de Investigación dependientes del Rectorado.

\subsection{En el aspecto normativo:}

Los siguientes son los documentos normativos básicos aprobados por el CU. definiendo el estatuto de la URP e instalando el VRI.

- $\quad$ El Reglamento General de investigación de la URP

- $\quad$ El Reglamento de Organización y Funciones del Vicerrectorado de Investigación.

- $\quad$ La Política General de Investigación

- $\quad$ Las Líneas de investigación de la Universidad Ricardo Palma

- $\quad$ El código de ética para la investigación científica tecnológica y humanística

- $\quad$ Las Normas Generales de Propiedad intelectual del docente investigador en la 
Universidad Ricardo Palma

- $\quad$ Las Normas para la publicación de Tesis en Cybertesis-URP

\subsection{En cuanto a fuentes de Financiamiento}

EI VRI maneja dos presupuestos anualmente:

- $\quad$ El presupuesto del Programa Anual de Investigación, que cubre los distintos proyectos de investigación presentados por los investigadores anualmente.

- El Fondo de Investigación para fines de apoyo al investigador docente y alumnos para que participen en certámenes nacionales e internacionales, para publicaciones e investigaciones especializadas, etc.

\subsection{En relación a la capacitación al Docente URP en investigación}

Desde el año 2016 se han realizado las siguientes actividades de capacitación:

- I y || Programa de especialización en investigación científica, tecnológica y humanística (2016)

- Curso Taller, Las revistas de investigación y los sistemas de indexación internacional (2016).

- Curso Taller: El ensayo académico y el artículo científico en las revistas de investigación con indexación internacional (2017).

\subsection{Proyectos de Investigación}

Constituido el VRI en el año 2016 se presentaron 43 proyectos de investigación por parte de los docentes de las distintas Facultades de la URP.

En el año 2017 se han presentado y sido aprobados 59 proyectos de investigación. Ambas cifras son bastante distantes de los proyectos de investigación que se presentaban antes de la creación del VRI tal como muestra el gráfico 1 que presenta los proyectos aprobados entre los años 2007 y 2017.

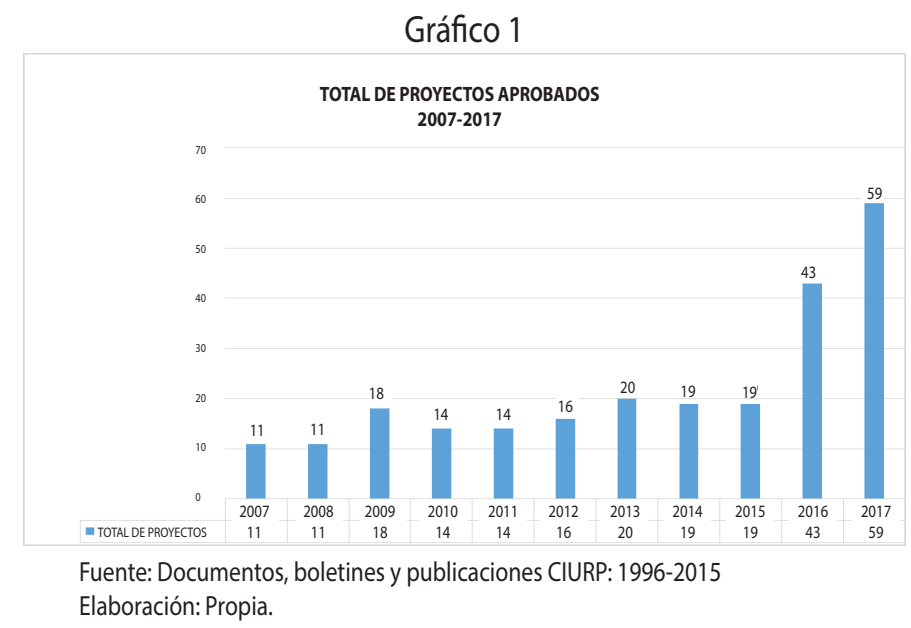

Además, se ha apoyado proyectos de investigación de tesis tanto para el pregrado como para el posgrado. En el año 2016 se apoyaron 6 proyectos de investigación. Para el 2017 se han hecho las convocatorias respectivas. 


\subsection{Publicaciones}

\section{a) Revistas}

Entre las publicaciones que más destacan son aquellas que están indexadas. La indexación se viene logrando a partir del año 2016 y son las siguientes:

- Revista Scientia del CIURP.

- $\quad$ La Revista de la Facultad de Medicina Humana.

- $\quad$ Revista Illapa del Instituto de Investigaciones museológicas.

- Revista de la Facultad de Humanidades y Lenguas Modernas

- Revista Aula Palma del Instituto Ricardo Palma.

- Revista Tradición, del Rectorado de la URP.

Están en proceso de indexación 4 revistas correspondientes al Programa de Estudios Básicos, Departamento de Humanidades, Facultad de Derecho y Ciencia Política y Facultad de Ingeniería.

\section{b) Cuadernos de Investigación.}

A partir del año 2017 se creó la publicación de la Serie Cuadernos de Investigación, que presenta importantes investigaciones realizadas por la URP. Se tiene dos publicaciones logradas.

№ 01, Violencia contra la mujer en el distrito de Santiago de Surco-Lima, Facultad de Derecho y Ciencia Política (2017).

$N^{0}$ 02, Test de Comprensión numérica para alumnos del nivel de Educación Primaria. Desarrollado por la Facultad de Psicología de la URP (2017).

Está en preparación el № 03 sobre Investigaciones Tecnológicas, realizado por la Facultad de Ingeniería de la URP.

\section{c) Boletín de Investigación}

Es una publicación del Vicerrectorado de Investigación de carácter informativo. Se viene publicando desde el año 2016 y hasta la fecha se han publicado 5 números.

\section{d) Catálogo de investigaciones publicadas URP 2000-216}

Publicado en mayo del 2017. Contiene en catálogo 50 publicaciones realizadas por la Editorial Universitaria y las Facultades de la URP entre los años 2000 al 2016. Destacan temas históricos, arquitectónicos arqueológicos, lingüísticos, psicológicos, educativos, culturales, etnográficos, y filosóficos entre los más importantes.

\section{e) Investigación formativa}

A partir del año 2016 se viene promoviendo las actividades y logros de investigación formativa en todas las facultades. En la primera Jornada de Investigación 2016 destacaron las Facultades de Psicología, Ciencias Económicas y Empresariales y la Facultad de Humanidades y Lenguas Modernas.

\section{f) Publicaciones internas}

Son publicaciones de tiraje corto. Se publican como documentos de trabajo. Hasta la fecha se han elaborado los siguientes:

- Guía Abreviada: Estilos de Redacción APA, Chicago y Vancouver

Dr. William Torres Acuña.

- Guía Resumida para Publicar Artículos de Investigación en Revistas Indizadas

Dr. Arnaldo Sánchez Bustillos. 
- El Ensayo Académico y el Artículo Científico en las Revistas de Investigación con Indexación Internacional. I y II Parte.

Mg. Saby Lazarte Oyague.

- El Artículo Científico en Revistas Médicas de Investigación Internacional Dr. Jhony A. De la Cruz.

- El Derecho de Propiedad Intelectual y Patente en el ámbito Universitario Dr. Carlos Sebastián Calvo.

- El modelo de Informe final de Investigación Lic. Patricia Matos Ramírez

- La Investigación Formativa en la Universidad Hugo Sánchez C., Jhony De la Cruz, Katia Mejía, Carlos Reyes R.

\subsection{Artículos académicos y de investigación}

Semestralmente el VRI solicita a los profesores investigadores y a los profesores a TC. que presenten un artículo científico o ensayo académico de acuerdo con los esquemas de la APA, Vancouver o Chicago. En el año 2016 se presentaron 54 artículos y en el primer semestre del presente año, igualmente se han presentando 54 artículos. Se revisa cada artículo y se logra seleccionar a los mejores para su ubicación en el Repositorio de Investigación.

\subsection{Investigadores en DINA Y REGINA}

A la fecha hay 266 docentes investigadores de la URP inscritos en DINA-CONCYTEC y 16 investigadores registrados en REGINA-CONCYTEC. Hasta fines del 2016 había 45 docentes investigadores registrados en la SUNEDU.

\subsection{Concursos convocados}

En el presente año se han convocado los siguientes concursos:

- Programa de incentivos al docente investigador de la URP que publica artículos en Revistas indizadas.

- I Concurso sobre Proyectos de Innovación Tecnológica para estudiantes de la Universidad Ricardo Palma

- Concurso de Proyectos de Tesis para pre grado y Pos grado. Convocado en el año 2016 y 2017.

\subsection{Repositorio Institucional y de investigación.}

El VRI en el 2016 creó el Repositorio de Investigación que se puede encontrar en la página web de la URP donde aparecen los principales logros y publicaciones realizadas por la Universidad en cuanto a Investigación.

Posteriormente se creó el Repositorio Institucional de la URP que contiene toda la información académica y de investigación de la URP

\subsection{Jornada de investigación}

Desde el año 2016 se instituyó el mes de la investigación en la URP y se definió la semana de investigación. El año 2016 se llevó a cabo entre el 17 y 28 de octubre del 2016 y el presente año se realizará la segunda semana del mes de octubre. Participan docentes investigadores, las Unidades de investigación de las Facultades y la Escuela de Posgrado, Los institutos especializados de investigación. Los resultados y logros de la I Jornada se publicaron en el Boletín №3 del VRI en diciembre del 2016. 


\section{Propuesta de indicadores para evaluar la investigación en la universidad}

Finalizamos esta exposición de logros presentando de manera sucinta una propuesta de 14 indicadores que pueden servir de base para fijar estándares mínimos que permitan indicadores de calidad y, que admitan evaluar el logro de una gestión en el campo de la investigación universitaria en nuestro país a fin de que puedan ser reconocidas nacional e internacionalmente.

a) Cantidad de centros, institutos y unidades de investigación con que cuenta la universidad.

b) Cantidad de los proyectos de investigación ejecutados anualmente considerando los campos (Científico, tecnológico y humanístico).

c) Cantidad de proyectos o estudios realizados considerando tipos de investigación (Básicos o aplicados)

d) Contribución teórico-práctica, beneficios o utilidad de los logros o resultados de las investigaciones.

e) Número de publicaciones específicas de las investigaciones realizadas anualmente.

f) Número de revistas de investigación indexadas publicadas anualmente.

g) Número de artículos de investigación en revistas indexadas anualmente.

h) Cantidad de tesis realizadas en pre y posgrado anualmente.

i) Cantidad de magister y doctores graduados en la universidad.

j) Número de investigadores en la Universidad.

k) Producción intelectual de los docentes investigadores.

l) Capacitación de los investigadores, cursos y programas de especialización.

m) Número de investigadores reconocidos por una entidad acreditadora.

n) Monto anual asignado a la investigación considerando el presupuesto global de la Universidad.

\section{REFERENCIAS BIBLIOGRÁFICAS}

Marticorena, B. (2016). Los desafíos de la investigación en el Perú. Edu-PUCP. www/puntoedu. pucp.edu.pe/.

Sánchez, C. H. (2015). Metodología y diseños en la investigación científica. Lima: Business Support Aneth.

Sánchez, C. H. (2016). Marco referencial para definir una política de investigación en las universidades. Lima: URP.

Sánchez, H. (2016). Desafios para la investigación en la Universidad Peruana. Boletin № 02 Agosto 2016

\section{Documentos consultados}

CONCYTEC-DINA.

Decreto Ley No 17437

Ley Universitaria $N^{\circ} 23733$

Ley Universitaria No 30220

Boletín del CIURP 1996 Universidad Ricardo Palma. 